AUTHOR CORRECTION OPEN

\title{
Author Correction: High-dimensional detection of imaging response to treatment in multiple sclerosis
}

Baris Kanber ${ }^{1,2,3}$, Parashkev Nachev ${ }^{3,4}$, Frederik Barkhof ${ }^{1,2,3,5}$, Alberto Calvi ${ }^{2}$, Jorge Cardoso ${ }^{6}$, Rosa Cortese ${ }^{2}$, Ferran Prados ${ }^{1,2}$, Carole H. Sudre ${ }^{6}$, Carmen Tur $\mathbb{D}^{2}$, Sebastien Ourselin ${ }^{6}$ and Olga Ciccarelli ${ }^{2,3}$

npj Digital Medicine (2019)2:66; https://doi.org/10.1038/s41746-019-0144-7

Correction to: npj Digital Medicine https://doi.org/10.1038/s41746019-0127-8, published online 10 June 2019

The original version of the published Article listed the incorrect affiliations for Jorge Cardoso, Carole H. Sudre, and Sebastien Ourselin. These authors' affiliations are now correctly noted as School of Biomedical Engineering \& Imaging Sciences, King's College London, London, UK. Additionally, a statement from the Acknowledgments section was omitted. The Acknowledgments have been updated to include the following: "We are grateful to the MS Society for its support of the Multiple Sclerosis Research Centre at the Queen Square Institute of Neurology where this work was completed." The HTML and PDF versions of the Article have been corrected.

\begin{abstract}
(i) Open Access This article is licensed under a Creative Commons Attribution 4.0 International License, which permits use, sharing, adaptation, distribution and reproduction in any medium or format, as long as you give appropriate credit to the original author(s) and the source, provide a link to the Creative Commons license, and indicate if changes were made. The images or other third party material in this article are included in the article's Creative Commons license, unless indicated otherwise in a credit line to the material. If material is not included in the article's Creative Commons license and your intended use is not permitted by statutory regulation or exceeds the permitted use, you will need to obtain permission directly from the copyright holder. To view a copy of this license, visit http://creativecommons. org/licenses/by/4.0/.
\end{abstract}

(c) The Author(s) 2019

\footnotetext{
${ }^{1}$ Centre for Medical Image Computing, Department of Medical Physics and Biomedical Engineering, University College London, London, UK; ${ }^{2}$ Multiple Sclerosis Research Centre, NMR Research Unit, Department of Neuroinflammation, Queen Square Institute of Neurology, University College London, London, UK; ${ }^{3}$ National Institute for Health Research (NIHR), University College London Hospitals Biomedical Research Centre (BRC), London, UK; ${ }^{4}$ High Dimensional Neurology Group, Queen Square Institute of Neurology, University College London, London, UK; ${ }^{5}$ Department of Radiology \& Nuclear Medicine, VU University Medical Center, Amsterdam, The Netherlands and ${ }^{6}$ School of Biomedical Engineering \& Imaging Sciences, King's College London, London, UK Correspondence: Parashkev Nachev (p.nachev@ucl.ac.uk)

These authors contributed equally: Baris Kanber, Parashkev Nachev

These authors jointly supervised this work: Sebastien Ourselin, Olga Ciccarelli
}

Published online: 16 July 2019 